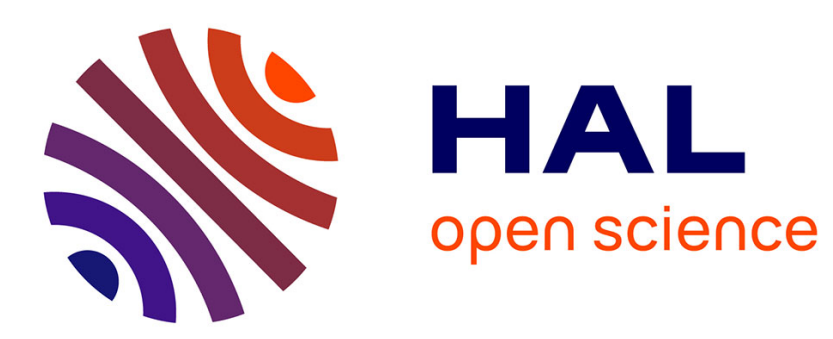

\title{
Molecular mechanics investigations of carbon nanotube and graphene sheet interaction
}

Mahamadou Seydou, Sophie Marsaudon, Julien Buchoux, Jean Pierre Aimé, Anne-Marie Bonnot

\section{- To cite this version:}

Mahamadou Seydou, Sophie Marsaudon, Julien Buchoux, Jean Pierre Aimé, Anne-Marie Bonnot. Molecular mechanics investigations of carbon nanotube and graphene sheet interaction. Physical Review B: Condensed Matter and Materials Physics (1998-2015), 2009, 80, pp.245421. 10.1103/PhysRevB.80.245421 . hal-01075297

\section{HAL Id: hal-01075297 https://hal.science/hal-01075297}

Submitted on 21 Oct 2014

HAL is a multi-disciplinary open access archive for the deposit and dissemination of scientific research documents, whether they are published or not. The documents may come from teaching and research institutions in France or abroad, or from public or private research centers.
L'archive ouverte pluridisciplinaire HAL, est destinée au dépôt et à la diffusion de documents scientifiques de niveau recherche, publiés ou non, émanant des établissements d'enseignement et de recherche français ou étrangers, des laboratoires publics ou privés. 


\title{
Molecular mechanics investigations of carbon nanotube and graphene sheet interaction
}

\author{
Mahamadou Seydou, ${ }^{*}$ Sophie Marsaudon, Julien Buchoux, and Jean Pierre Aimé ${ }^{\dagger}$ \\ Centre de Physique Moléculaire et Optique Hertienne (CPMOH), Université de Bordeaux 1, 351 Cours de la Libération, \\ Talence 33400, France \\ Anne Marie Bonnot \\ Institut Neel, CNRS, 25 Avenue des Martyrs, BP 166, 38042 Grenoble Cedex 9, France \\ (Received 22 June 2009; revised manuscript received 8 September 2009; published 15 December 2009)
}

\begin{abstract}
The interaction between a carbon nanotube $(\mathrm{CNT})$ and a graphene sheet is investigated to describe the contact properties between a CNT atomic force microscope (AFM) tip and a graphite surface. The energy of the whole system is calculated using $\mathrm{MM}+$ molecular mechanical modeling. With the numerical calculations, one explores the sliding motion of the CNT on the graphene sheet either at the CNT apex or with a given CNT length contacting the surface. The aim is to mimic the AFM CNT tip scanning a graphite surface. To do so we calculate the energy barriers, the tips have to overcome to achieve a full translation. The results show that the barrier heights markedly depend on the contact length between the CNT and the graphene but show a weak dependence, if any, on the CNT tube diameter.
\end{abstract}

DOI: 10.1103/PhysRevB.80.245421

PACS number(s): 03.65.Sq, 02.60.Cb, 07.79.Lh, 02.70.Ns

\section{INTRODUCTION}

In the last decade carbon nanotubes (CNTs) have attracted great interest due to their mechanical and electronic properties. ${ }^{1-4}$ More specifically, their high tensile strength, flexible rigidity, high aspect ratio, and low chemical reactivity make them interesting materials for tips in scanning probe microscopy. Single-wall CNT tip scanning using dynamical AFM imaging has been demonstrated to provide singlemolecule profiles of a hydrated protein ${ }^{5}$ and DNA molecules 6 with enhanced resolution and reproducibility.

The design of CNT AFM tips (with, in particular, the nanotube attach to the surface, its angle with the surface) is determinant for the AFM image contrast; it depends on the nanotube substrate interaction, thus on the geometry of the contact area between the CNT and the surface. Due to the weak chemical affinity of CNT, AFM experimental studies with CNT AFM tips, show remarkable tip and image stability but many years after early research on the nonlinear behavior of single-wall probes, ${ }^{7}$ a quantitative analysis still remains a major challenge.

Experimental measurements are usually done on natural graphite, which is a mixture of ordered $A B$ stacked and disordered turbostatic layered structures, ${ }^{8}$ shown in Fig. 3. The equilibrium interlayer distance ${ }^{9} \quad 3.34 \AA$ and $c$-axis compressibility $^{10}\left(2.7 \times 10^{-12} \mathrm{~cm}^{2} / \mathrm{dyn}\right)$ of graphite can be measured with good accuracy. The absolute interlayer binding energy, i.e., exfoliation energy is harder to extract; there is only a handful of results in the literature. A heat-of-wetting experiment by Girifalco and Ladd ${ }^{11}$ gives an exfoliation energy of $43 \mathrm{meV} /$ atom. A microscopic determination of the interlayer binding through study of collapsed nanotubes yields $35 \pm 10 \mathrm{meV} /$ atom. $^{12}$ Later experiments on thermal desorption of polyaromatic hydrocarbons give a larger binding energy of $52 \pm 5 \mathrm{meV} /$ atom. $^{13}$ Concerning interaction between carbon nanotube walls, Kis et al. ${ }^{14}$ had performed interlayer force measurements through cyclic telescoping motion of a multiwall carbon nanotube. They showed that the force acting between the core and the outer casing is modulated by the presence of stable defects and generally exhibits ultralow friction, below the measurement limit of $-1.4 \times 10^{-15} \mathrm{~N} /$ atom and total dissipation per cycle lower than $0.4 \mathrm{meV} /$ atom.

To interpret these experimental values, some theoretical calculations have been carried out on graphene interlayer spacing and binding energy. Trickey et al., ${ }^{15}$ in 1992 performed density-functional theory-local-density approximation (DFT-LDA) studies of the binding between two graphene sheets. They obtained an interlayer binding energy that was $50 \%$ larger than the experimental ones. Also, an LDA calculation by Jansen and Freeman ${ }^{16}$ gave a fairly good answer for the interlayer spacing $3.41 \AA$ but the interlayer binding energy was four times greater than the expected value. The authors explain the difference because of a local density that does not adequately take into account the dispersive part of the van der Waals interactions. Using the localdensity approximation to DFT, within pseudopotential formalism and plane-wave basis, Schabel and Martins, ${ }^{17}$ found an interlayer spacing and binding energy in excellent agreement with the experimental results. Girifalco and Hodak ${ }^{18}$ used an empirical model potential and obtained an interlayer and binding energy also in good agreement with the experiment. In the other hand, Charlier et al. ${ }^{8}$ identified various contributions to the interlayer binding and gave the correct interlayer spacing and binding energy.

Other theoretical studies have been dedicated to interaction between carbon nanotube. Using quantum mechanical augmented by an intertube Lennard-Jones potential, Surján and co-workers ${ }^{19}$ have computed interactions of several families of tubes of diameters. Depending on the stacking between tubes, they obtained a rotation barrier in the range 3.9-29.3 $\mathrm{meV} / \AA$. Density-functional theory within the local-density approximation has been used to compute the translation and rotation barrier of two concentric bilayered carbon nanotubes. ${ }^{20}$ The results gave a translation and rotation barrier equal to 0.23 and $0.52 \mathrm{meV} / \AA$, respectively.

In recent experiments and theoritical studies, Rao and coworkers, demonstrated that graphene samples prepared by 


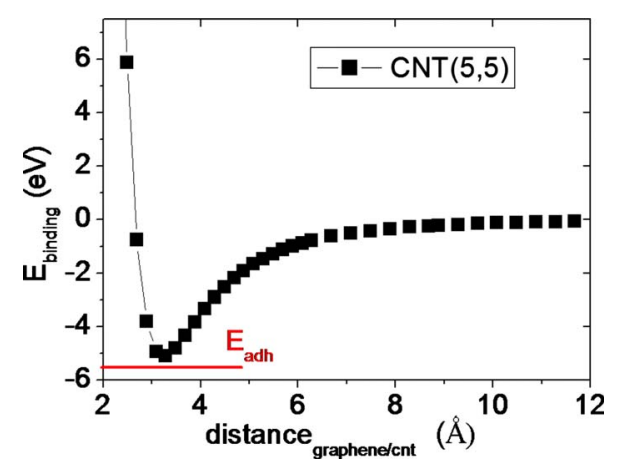

FIG. 1. (Color online) Binding-energy calculation for carbon nanotube. Adhesive energy $E_{a d h}$ is the lowest value of binding energy.

exfoliation of graphitic oxide or conversion of nanodiamond exhibit significant uptake of $\mathrm{H}_{2}$ and $\mathrm{CO}_{2} \cdot{ }^{21}$ They used DFT calculations with localized orbitals implemented in SIESTA software to interpret their experiments, and obtained a binding energy of $-0.21 \mathrm{eV}$ for $\mathrm{H}_{2}$ and $-0.4 \mathrm{eV}$ for carbon dioxide. In another paper they also studied binding of DNA nucleobases and nucleosides with graphene. ${ }^{22}$ The total binding energy obtained by adding the van der Waals term, found thus are $-0.65,-0.64,-0.638$, and $-0.34 \mathrm{eV}$ for Guanine, Adenine, Thymine, and Cytosine, respectively.

Here, we perform calculation of the total energy involved in a CNT-graphene sheet ensemble, with the aim to describe the CNT tips behavior when it scanned on a graphite surface, by using $\mathrm{MM}+$ force field. The MM+ force field is used to calculate the interaction between aromatics molecules. ${ }^{23}$ For instance, Ianelli et al. ${ }^{24}$ have systematically compared the numerical results obtained with different force-field method with the x-ray structural data dedicated to a study of the influence of the packing forces on the molecular conformation of complex molecules in the solid state. Among different molecular mechanics calculations, $\mathrm{MM}+$ provided better results. The same conclusion was reached in doing ansa-9meta-cyclophane studies. ${ }^{25} \mathrm{MM}+$ was also used to calculate various stables structures such as host alcene-octaamidocavitands@ $\mathrm{C}_{60}$ (Ref. 26) or biological systems. ${ }^{27}$ Concerning adsorption studies, MM+ calculations have been found as an appropriate tool to help the experimental finding. For instance, for the study of the deposition of pheniloctane on graphite surface, $\mathrm{MM}+$ gives numerical results in good agreement with the experimental data, in particular, on the relative orientation of the molecule on the graphite surface. ${ }^{28}$ It has been also widely used to account for scanning tunnel microscope (STM) measurements, MM + calculations on methionine adsorption onto the graphite surface have been carried out recently and have helped to find the exact site of adsorption of the molecule on graphite. ${ }^{29}$ Following the same aim, STM and MM+ calculations were employed to analyze the step of nanoassembly of meso-tetraphenylporphines H2TPP and it's cobalt (II) complex on the highly oriented pyrolitic graphite. ${ }^{30}$

Recent AFM thermal-noise measurements on CNT have shown that the contact between CNTs and a graphite surface can be classified in two categories: either the contact obeys a free sliding condition or else a pinned situation. ${ }^{31}$ As thermal

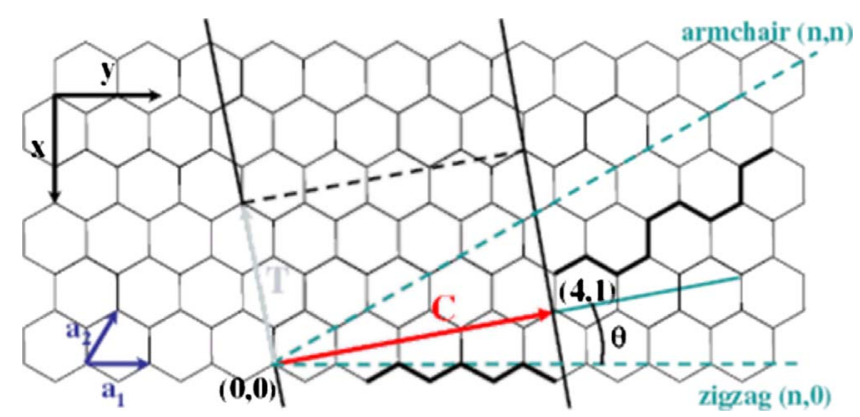

FIG. 2. (Color online) Building a SWNT by rolling a graphene sheet. The gray domain is the CNT surface, the straight black lines are the connecting lines closing the tube. The chiral vector $C$ is perpendicular to the tube axis $T$. The CNT is defined with the chiral indices $(n, m)$ and the units vectors $a_{1}$ and $a_{2}$. On the figure $C=4 a_{1}+a_{2}$. Particular values of the indices lead to nonchiral, $(n, n)$ armchair, and $(n, 0)$ zigzag $\mathrm{CNT}$.

noise applies a weak forcing, tiny variations can be observed, however the main question remains why similar CNTs may exhibit complete different contact behaviors. The present work aims at providing a step to understand such a difference, in particular, to analyze the parameters governing the height of the barrier that opposes to the CNT tip translation on the graphite.

\section{CALCULATION METHOD}

Molecular mechanics calculation were performed with the HYPERCHEM program employing the MM+ force field. It is a general force field improved from the MM2 force field first developed by Allinger. ${ }^{32,33}$ The system total energy is a sum of bond stretching, bond-angle binding, dihedral, improper dihedral, and van der Waals terms.

Unlike other force fields, MM+ describes the interaction of van der Waals by combining an exponential repulsion with an attractive $\frac{1}{R^{6}}$ dispersion interaction. So the van der Waals term is calculated as ${ }^{34}$

$$
E_{\text {van der Waals }}=\sum \varepsilon_{i j}\left[2.9 \times 10^{5} \exp \left(-12.5 \rho_{i j}\right)-2.25 \rho_{i j}^{-6}\right],
$$

where $\varepsilon_{i j}=\sqrt{\varepsilon_{i} \varepsilon_{j}}$ and the hardness parameters $\varepsilon_{i}$ determine the depth of the attractive well and are defined for a pair of atoms and $\rho_{i j}=\frac{R_{i j}}{r_{i j}^{*}}, r_{i j}^{*}=r_{i}^{*}+r_{j}^{*}, r_{i}^{*}$ is the van der Waals radius for the atom of type $i$ and $R_{i j}$ is the distance between the core of the two atoms.

Structures of CNT and graphene are built with the NANOTUBE MODELER software. ${ }^{35}$ In graphene structure the distance between neighboring carbon atoms is $1.41 \AA$. To do calculations, the default parameters from Goddard and co-workers ${ }^{36}$ are modified to graphitic systems. $r_{i j}^{*}$ is taken to be equal to $3.30 \AA$ and $\varepsilon_{i j}$ to $3.66 \mathrm{meV}$.

Single-walled CNTs, SWNT, of diameters ranging between 0.7 and $2.56 \mathrm{~nm}$ and indices $(6,0),(8,0),(9,0)$, and $(5,5),(6,6),(10,10),(20,20)$ corresponding to zigzag and armchair structures, respectively, (see Fig. 2) are used. The ends are opened for $(6,0),(8,0)$, and $(20,20)$ and capped for $(5,5),(6,6),(9,0)$, and $(10,10)$ CNTs. Within the range of 

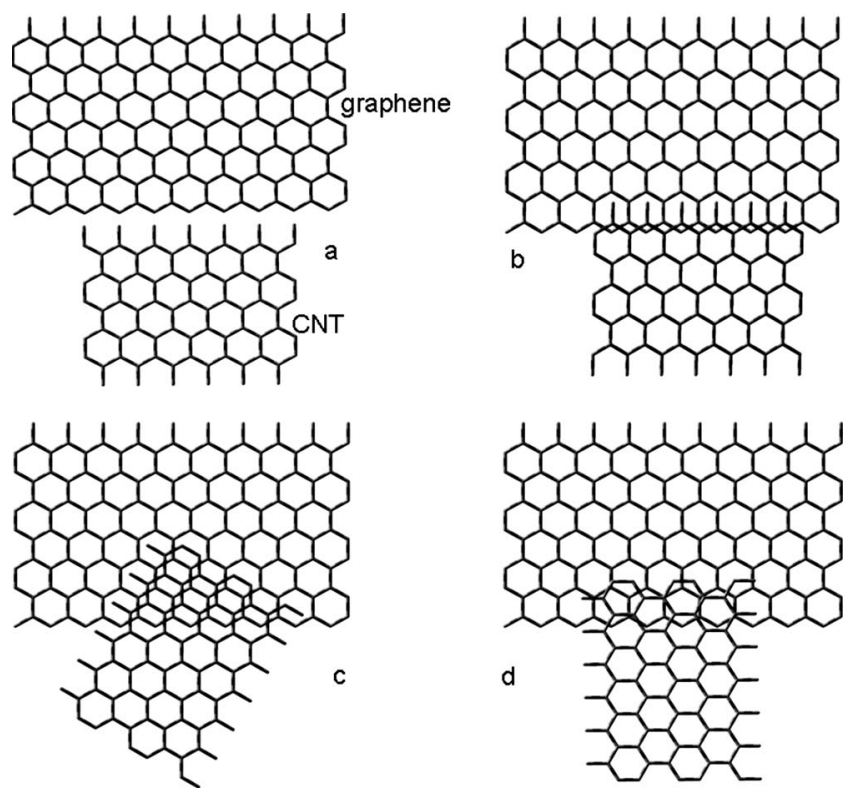

FIG. 3. (a) Sketch of the direction of the SWNT translation motions on the graphene. Two main stacking are usually considered; respectively, called $A A$ (perfect registry between the aromatic rings); $B B$ when one bond is set above the middle of the underlying aromatics rings [see figure (b)]. (b) Motion of a zigzag SWNT forming $A A$ and $A B$ stacking; (c) translation of a armchair SWNT with a fixed relative orientation of $60^{\circ}$, the translation can generate $A A$ and $A B$ structures; (d) $90^{\circ}$ rotation of the structure displayed in figure (b), the resulting system structure is called turbostatic stackings.

CNTs diameters, the relaxation does not change considerably $^{37,38}$ the structure of the tube so the energy is calculated without including a relaxation process. Also, no relaxation is applied on the graphene. The area is a large sheet of graphene $80 \AA \times 80 \AA$. The calculation is performed so that the smallest distance between the tube end and the edge of the graphene is greater than $1 \mathrm{~nm}$.

The method aims to calculate the binding energy of the system CNT-graphene sheet. Before this procedure, we calculate the variation in the energy along the $z$ axis by varying the distance between the graphene and the CNT from 15 to $2 \AA$ by step of $0.2 \AA$ (Fig. 1).
For each point we do a single-point calculation of the energy of the systems (CNT+graphene) and of the isolated CNT and graphene. The binding energy is obtained as the difference between the total energy of the system and the sum of the energy for each isolated parts (Fig. 1),

$$
E_{\text {binding }}=E_{\mathrm{CNT}+\text { graphene }}-\left(E_{\mathrm{CNT}}+E_{\text {graphene }}\right) .
$$

The binding energy depends only on the van der Waals part. The adhesive energy is taken as the lowest value of binding energy. To compute the barrier height, we explore the adhesive energy by moving the tube along its axis and diameter direction by step of $0.2 \AA$ (Fig. 4).

Folding of a graphene sheet along the vector $\vec{C}$ gives the carbon-nanotube structure, for instance, zigzag and armchair (see Fig. 2).

\section{RESULT AND DISCUSSIONS}

\section{Influence of the stacking structure}

To explore several types of contact between the carbon nanotube and the graphene, we first investigate armchair and zigzag structures deposited along the main axis, then rotate the CNT on the surface with $60^{\circ}$ and $90^{\circ}$ angles and optimize for each position the equilibrium distance. As showed in Fig. 3, several conformations are found. A translational motion is then performed along the direction of the tube symmetry axis with steps of size $0.2 \AA$.

The adhesion of the CNT on the graphene is defined as the lowest-energy value giving the equilibrium distance between the CNT and the graphene. For graphite interlayers the calculated distances are found between 2.8 and $4 \AA$ (Refs. $16,20,39$, and 40) while the equilibrium distances between the CNTs and the graphene sheet are found to be in between 3 and $3.2 \AA .^{41}$ In order to mimic the motion of a CNT AFM tip on the graphite, we first investigate the relative translation motion of a SWNTs of $2 \mathrm{~nm}$ length on graphene. The number of atoms per tube is 114 for $(6,0), 152$ for $(8,0), 204$ for $(9,0), 221$ for $(5,5), 252$ for $(6,6), 480$ for $(10,10)$, and 680 for $(20,20)$. The addressed question is to calculate the potential barrier we need that must be overcome to achieve such a lateral motion. Several configurations are envisioned and some of them are sketched in the Fig. 3. We first look at the
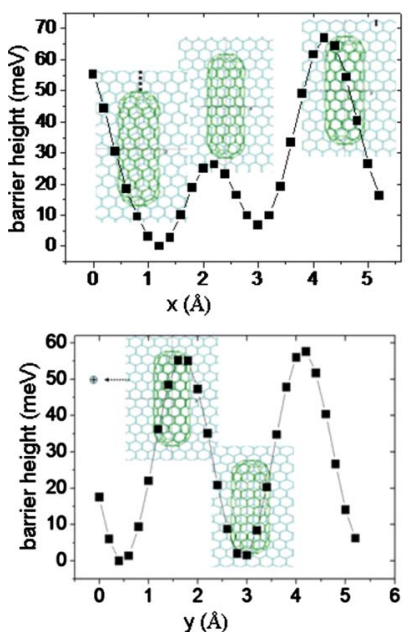

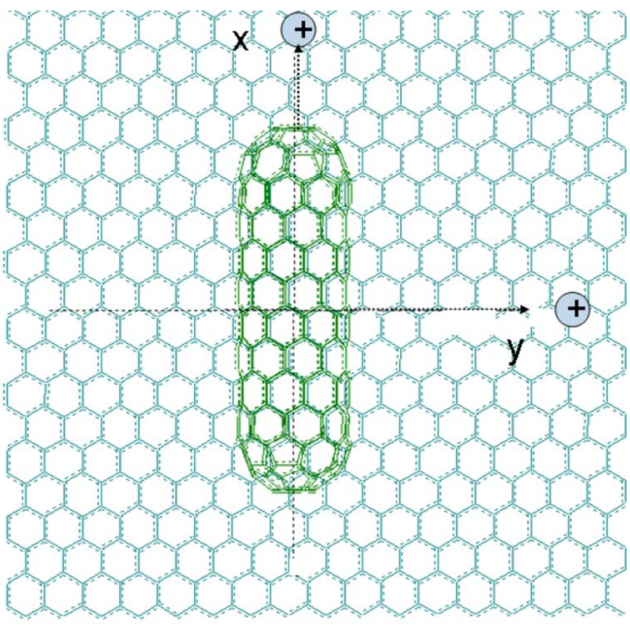

FIG. 4. (Color online) Barrier heights of the translation motion of the SWNT $(9,0)$ along the $x$ and $y$ axes. Left: scheme of the configuration investigated. Right graphs: energy barrier heights we need to overcome to achieve a full translation with the schemes of the different overlap structures between the SWNT and the graphene. Top motion in the nanotube axis and down motion in the diameter axis. 
TABLE I. Adhesive energy for CNTs, tube lengths are $2 \mathrm{~nm}$, number of atoms per tube is 204 for $(9,0), 221$ for $(5,5), 252$ for $(6,6)$, and 480 for $(10,10)$. Indices 1,2 , and 3 designate overlapping configurations $b, c$, and $d$ as displayed in Fig. 3 .

\begin{tabular}{ccccc}
\hline \hline CNT & $\begin{array}{c}E_{a d h} \min \\
(\mathrm{eV})\end{array}$ & $\begin{array}{c}E_{a d h} \max \\
(\mathrm{eV})\end{array}$ & $\begin{array}{c}\text { Barrier height } \\
(\mathrm{meV} / \text { atom })\end{array}$ & $\begin{array}{c}E_{a d h} \min \\
(\mathrm{meV} / \text { atom })\end{array}$ \\
\hline$(9,0)_{1}$ & -5.3941 & -5.3788 & 0.28 & -26.44 \\
$(9,0)_{2}$ & -4.9708 & -4.8983 & 0.26 & -24.36 \\
$(9,0)_{3}$ & -4.8705 & -4.7325 & 0.043 & -23.87 \\
$(5,5)_{1}$ & -5.16606 & -5.08865 & 0.35 & -23.40 \\
$(5,5)_{2}$ & -4.7900 & -4.7324 & 0.26 & -21.67 \\
$(6,6)$ & -5.3461 & -5.2894 & 0.22 & -21.21 \\
$(10,10)$ & -6.7190 & -6.6449 & 0.15 & -14.0 \\
\hline \hline
\end{tabular}

simple translation of a SWNT $(9,0)$ along $x$ and $y$ axes, where $x$ is along the tube-axis direction (Fig. 4).

In Fig. 4, the translation motion along the tube axis shows two barrier heights of 30 and $70 \mathrm{meV}$. For the second barrier, the SWNT starts from a local minimum of $7 \mathrm{meV}$. Therefore, the effective value of the second barrier height is reduced to about $63 \mathrm{meV}$. The lateral force required to pass over the barrier is readily obtained by dividing the distance between the neighboring local minimum and the maximum height of the barrier, which for the two cases is about $1 \AA$, we thus have 48 and $100 \mathrm{pN}$, respectively. The lateral motion along the $y$ direction exhibits two identical barrier heights of 57 $\mathrm{meV}$ with a distance between minimum and maximum of $1.2 \AA$. The corresponding lateral force is $76 \mathrm{pN}$. Note that the calculation investigating the relative rotation of a $(10,10)$ CNT against a fixed one, leads to similar shape of the barrier potential. ${ }^{19}$

The top of the barrier corresponds to a perfect superposition between the aromatic rings. On the contrary, at the location of the minimum of energy, the stacking is such that a carbon atom of the CNT is at the center of a benzene ring of graphene as those shown in Fig. 4(b). These structures are similar to those observed for graphite interlayer. ${ }^{41,42}$

Changes in the adhesive energy depend both on the CNT graphene relative orientation and on the CNT structure, either zigzag or armchair (see Table I). Therefore, we may also expect that the energy landscape and, in particular, the height

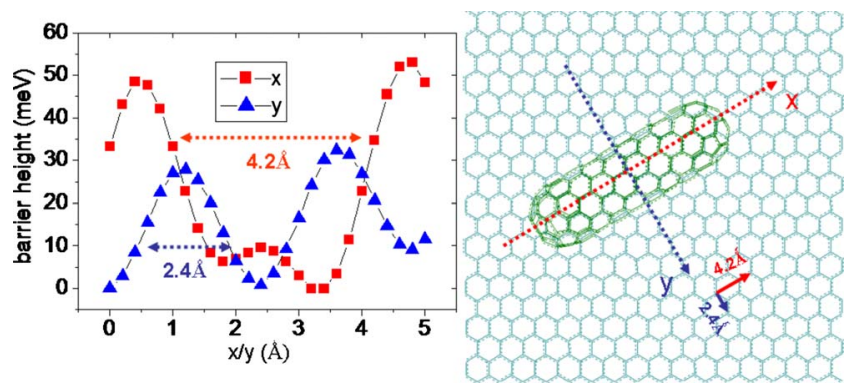

FIG. 5. (Color online) Height barrier for a $(9,0) \mathrm{CNT}$ rotated of $60^{\circ}$ as regard to the graphene. Scheme of the $(9,0)$ CNT rotated of $60^{\circ}$ as regard to the graphene potential periodicity linked graphene structure.

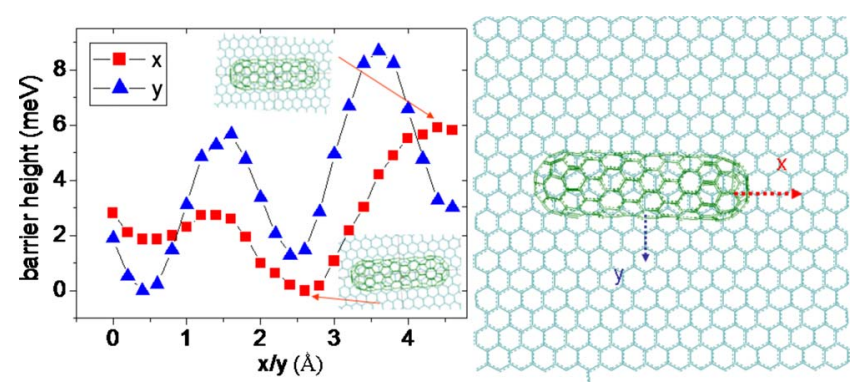

FIG. 6. (Color online) Sliding zigzag CNT on armchair direction of graphene.

of the barriers might also depends on the relative orientation between the two structures. As shown with the results displayed in Figs. 4-6 the energy landscape depends drastically on the relative orientation between the CNT and graphene. For instance, the $90^{\circ}$ rotation of the $(9,0)$ CNT modifies the shape of the barrier and leads to a net decrease in the barrier height, much less than $k_{B} T$, so that the tube may slide on the surface.

The $60^{\circ}$ rotation of the $(9,0) \mathrm{CNT}$ (Fig. 5) shows more complicate energy landscapes. The translation motion along the tube-axis direction exhibits a small bump of $10 \mathrm{meV}$ height and two barrier heights of values 50 and $57 \mathrm{meV}$ with the maxima being separated by a distance of $4.2 \AA$. The lateral displacement along a direction perpendicular to the CNT axis exhibits symmetric barriers of $30 \mathrm{meV}$ heights. Those values are close to the room-temperature value 25 meV.

When the CNT is rotated by $90^{\circ}$, the barrier heights are drastically reduced with heights much lower than the roomtemperature energy. The result is obtained for the two directions, for translation along the tube axis $(x)$ or perpendicular to it $(y)$ (Fig. 6). Those results suggest that the tube may exhibit large fluctuations of the contact area with the graphene.

\section{EFFECT OF THE TUBE DIAMETER ON THE BARRIER HEIGHT AS THE CNT SLIDES}

For the armchair tubes $(5,5)$ and $(6,6)$ (Fig. 7), the energy landscapes are similar to the one obtained with the zigzag structure. The translation motion along the tube axis $(x \mathrm{di}-$ rection) gives a set of barrier heights and periodicity similar to those corresponding to the translation motion along the direction normal to the $(9,0)$ zigzag structure (along the $y$
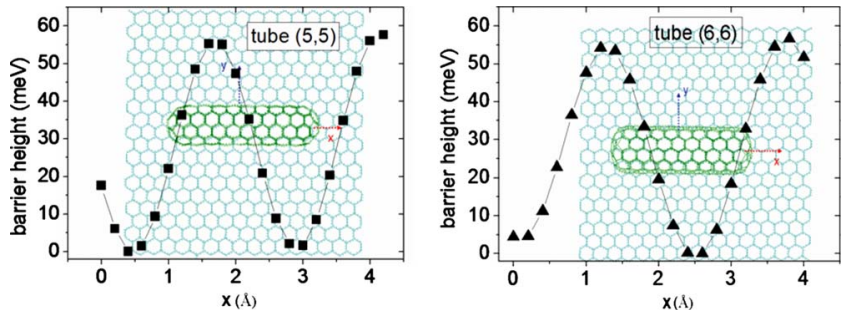

FIG. 7. (Color online) Barrier-height variation with $x$ displacement for tube $(5,5)$ (left) and $(6,6)$ (right). 

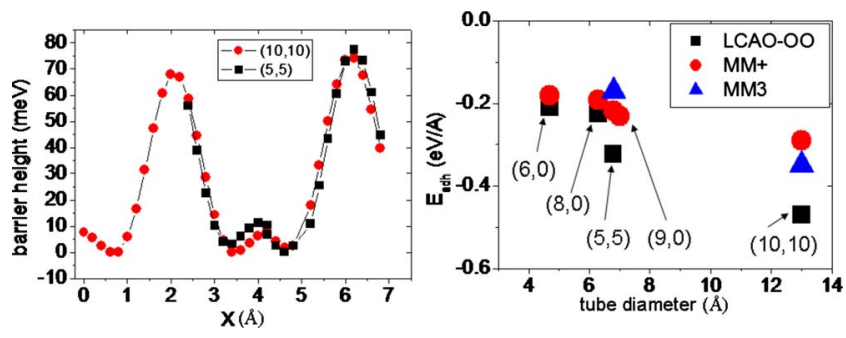

FIG. 8. (Color online) Left, comparison of barrier heights for CNTs $(5,5)$ and $(10,10)$ (221 and 480 atoms, respectively). The shape of the barriers are nearly the same exhibiting a weak dependence on the tube diameter if any. Right graph, diameter dependence of adhesive energy computed with MM+, MM3 (Ref. 37), and LCAO-OO (Ref. 45).

direction in Fig. 4). A similar behavior is also obtained between a lateral motion along the $y$ direction, armchair structure, and along the $x$ direction for the zigzag structure (results not shown). The increase in the diameter from $(5,5)$ to $(6,6)$, leads to an increase in the adhesive energy from -5.16 to $-5.34 \mathrm{eV}$. However, the barrier heights remain nearly equal with a value of $57 \mathrm{meV}$ (Fig. 8). Whatever the involved translational motion involved, along the tube axis or perpendicular to it, the same conclusion is valid for the zigzag and armchair structures with barrier heights often higher than $k_{B} T=25 \mathrm{meV}$. The energy landscape of $(5,5)$ and $(6,6)$ structures show a weak dependence, if any, on the CNT diameter. This result is confirmed with the comparison of barriers heights for the $(5,5)$ and $(10,10)$ structures (Fig. 8).

\section{INVESTIGATION OF THE EFFECT OF THE CNT CONTACT LENGTH ON THE RELATIVE MOTION BETWEEN CNT AND GRAPHENE}

As expected, an increase in the contact length of the tube on the graphene leads to a corresponding increase in the adhesive energy. But also, the barrier heights are augmented with 35,45 , and $55 \mathrm{meV}$ for $1,1.5$, and $2 \mathrm{~nm}$ of contact CNT length, respectively (Fig. 9).

In the Table I, we report the adhesive energy and barrier height per atom calculated for the CNT structures $(9,0)$, $(5,5),(6,6)$, and $(10,10)$. The adhesive energies per atom are

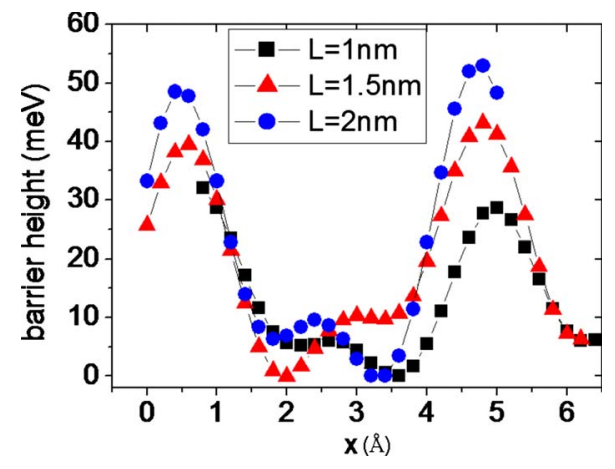

FIG. 9. (Color online) Variation in adhesive-energy barrier with the length of the CNT $(9,0)$.
TABLE II. Adhesive energy for different lengths of CNT $(9,0)$, with 132,186 , and 204 atoms, respectively, for $1,1.5$, and $2 \mathrm{~nm}$.

\begin{tabular}{ccc}
\hline \hline $\begin{array}{c}\text { Length } \\
(\mathrm{nm})\end{array}$ & $\begin{array}{c}E_{\text {adh }} \min \\
(\mathrm{eV})\end{array}$ & $\begin{array}{c}E_{\text {adh }} \min \\
(\mathrm{meV} / \mathrm{atom})\end{array}$ \\
\hline 2 & -4.9708 & -24.36 \\
1.5 & -3.688 & -19.82 \\
1 & -2.802 & -21.22 \\
\hline \hline
\end{tabular}

within the range 14-26.14 meV/atom, in good agreement with the experiment values from Ref. 12 .

In Fig. 8, adhesive energies of CNTs $(6,0),(8,0),(9,0)$, $(5,5),(6,6),(10,10)$, and $(20,20)$ are presented. Values are, respectively, $-0.18,-0.19,-0.26,-0.25,-0.33$, and $-0.40 \mathrm{eV} / \AA$. The equilibrium distances are within the range 3.0-3.2 $\AA$. The results are in a good agreement with Avouris et al. results. ${ }^{37}$ and $\mathrm{C}_{60}$ adsorption on graphite results. ${ }^{43}$ They are slightly larger then the interlayer interactions energies of graphite calculated with Moller-Plesset second-order method (MP2/6-31G $\left.\mathrm{G}^{*}\right){ }^{42}$ To evaluate the effect of the electronic energy, we compare our results to DFT/LCAO-OO+intermolecular perturbation theory (used to add van der Waals contribution). ${ }^{38,44}$ Calculations have been realized on the same system with the same method to compute the adhesive energy. ${ }^{45}$ We obtain some differences in the adhesive energy depend on the diameter. The difference is very small for small diameters and becomes significant up to $0.2 \mathrm{eV} / \AA$ for the nanotube $(10,10)$. This difference is due to the electronic energy contribution from atomic orbitals overlapping, which becomes more important with increasing number of atoms of CNT interacting with the surface because of the tube deformation.

Less obvious are the variations in the energy landscape, the barrier heights show a marked increase as a function of the contact length with 35,45 , and $55 \mathrm{meV}$ for $1,1.5$, and 2 nm of contact CNT length, respectively (Fig. 9) (Table II).

Previous results ${ }^{19}$ focusing on the interaction energies per length for aligned $\mathrm{CNT}$ pairs give barriers in the range 26-33.1 meV/ $\AA$ for $A A$ and $B B$ structures overlap and much less $(4.2-8 \mathrm{meV} / \AA$ ) for turbostatic structures (see Fig. 3 for the definition of the structures). These results are larger than those we have displayed in Figs. 4-6 because of the strong bind between tubes and the rotation effect which include an important contribution of orbital overlapping. Our results agree quite well with the experimental CNTs measurements ${ }^{46}$ investigating the sliding motion betwen two concentric CNTs, and density-functional theory within LDA approximation of the same system where an estimate of the translation and rotation barriers are, respectively, 0.23 and $0.52 \mathrm{meV} / \mathrm{atom}$ (Ref. 20) (see Table I).

\section{MIMICKING AFM CNT TIP SCANNING A GRAPHENE SURFACE}

The above methodology might help to simulate the behavior of a CNT AFM tip touching and moving on a graphite surface. Here, only the energy of adhesion is taken into ac- 

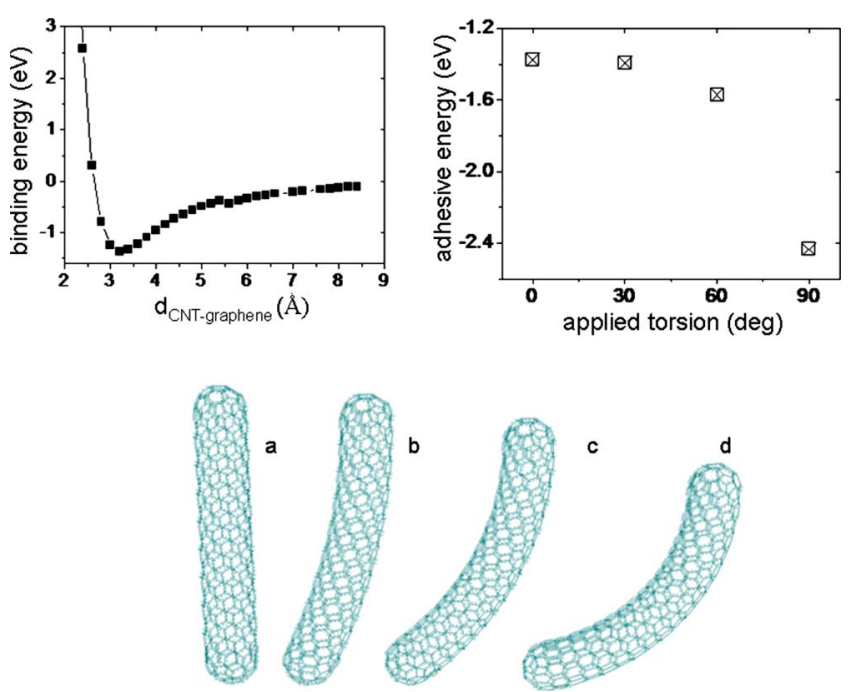

FIG. 10. (Color online) Top: left, binding-energy variation in $(9,0)$ CNT without torsion, right, adhesive energy in function of torsion angle. Bottom, structures of $\operatorname{CNT}(9,0)$ without torsion (a), with torsion of $30^{\circ}$ (b), $60^{\circ}$ (c), and $90^{\circ}$ (d). Structures are built with NANOTUBE MODELER software.

count in the following discussion. To do so, we choose a CNT $(9,0)$ with a length of $4 \mathrm{~nm}$ and a diameter of $0.7 \mathrm{~nm}$. The graphene is a rectangular surface with a size of $3 \mathrm{~nm}$ per $9 \mathrm{~nm}$. All apex of CNT are capped. we investigate some situations: a first one, where the tube interacts with the graphene area without any torsion so that the tube is perpendicular to the surface; other ones, where the tube is undergoes a torsion of $30^{\circ}, 60^{\circ}$, and $90^{\circ}$ (Fig. 10) so that more CNT atoms are interacting with the surface.

The structures of the CNT constraint and the graphene are built with the NANOTUBE MODELER software. ${ }^{35}$ In an attempt to be as close as possible to the experimental situations, the binding energy is calculated along the $z$ axis by approaching the CNT and searching the lowest interacting energy at equilibrium distance (Fig. 4). Variation in binding energy of CNT without torsion is reported in Fig. 10 and show an adhesive energy of $-1.37 \mathrm{eV}$ at an equilibrium distance of $3.2 \AA$. In the same figure adhesive-energy variation in function of the applied torsion is presented. The results show an adhesive energy around $-1.37,-1.39,-1.57$, and $-2.43 \mathrm{eV}$, respectively, for the CNT without and with $30^{\circ}, 60^{\circ}$, and $90^{\circ}$ torsion. As indicated with the values reported in the Fig. 10, the increase in binding energy may be directly connected to an increase in the contact length for the bending CNT. For the tube without any torsion, the adhesive energy is less than the value calculated for the fullerene. ${ }^{38,43}$ This difference can be explained by the difference between the CNT $(9,0)$ and the fullerene diameter size and an additional electrons overlap contribution in DFT.

Then, the minimum energy being reached, a lateral displacement of the CNT is started that corresponds to the $x$ axis, as shown in Fig. 10, when the CNT is perpendicular to the surface or along the direction nearly parallel to the CNT axis for the CNT under torsion. The incremental step motion is $0.2 \AA$. The CNT without torsion gives a barrier energy of $13.14 \mathrm{meV}$, for a distance of $1.4 \AA$ between the local mini-

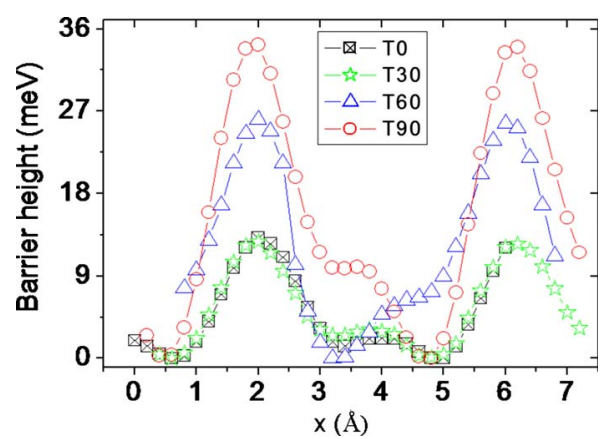

FIG. 11. (Color online) Variation in adhesive energy by dragging the CNT $(9,0)$ without torsion (rectangle) and with torsion $30^{\circ}$ (star), $60^{\circ}$ (triangle) and $90^{\circ}$ (circle) in $x$ direction.

mum and maximum. So that, only a lateral force as low as $15.31 \mathrm{pN}$ is required to induce the lateral displacement. The barrier energy is almost half of the thermal energy $k_{B} T \sim 25 \mathrm{meV}$ at $T=300 \mathrm{~K}$ and the tube can slide freely on the graphene.

For the twisted CNT, the barrier heights are, respectively, $12.75,26.1$, and $34.23 \mathrm{meV}$ for $30^{\circ}, 60^{\circ}$, and $90^{\circ}$ torsion (see Fig. 11) with a lateral displacement of $1.4 \AA$ leading to a lateral force of $14.56,29.82$, and $39.2 \mathrm{pN}$ to pass over the barriers. Therefore, as soon as the CNT increases its contact length, it can become trapped in some areas. Both free sliding and pinning of AFM CNT tips ${ }^{47-49}$ have been observed experimentally on a graphite surface using thermal-noise measurements. ${ }^{31}$ As shown in this work, these situations can be explained by a possible increase in the contact length together with a more or less good registry between the CNT and the graphite.

\section{CONCLUSION}

In this paper, we have calculated the contact interaction between various carbon nanotubes and a graphene surface. The numerical calculations are used to mimic the lateral motion of a CNT AFM tip on a graphite surface. We show that the barrier heights of the lateral motion energy landscape depend very weakly on the tube diameter while it strongly varies with the contact length of the CNT on the surface. Also the barrier heights strongly depend on the relative orientation between the tube and the surface. Therefore the nanotube can slide freely or be trapped at room temperature, depending on how the contact between the CNT and the surface takes place. The bending of a CNT on a surface increases the interaction and the height of the barrier. For applications in AFM, our numerical results show how much the lateral displacement of the CNT AFM tip depends on the contact length between the CNT and the surface and of their relative orientation, thus the angle of the contact with the surface.

\section{ACKNOWLEDGMENTS}

We thank the ANR P3N for funding the research with the program IMPROVE-LM. It is a pleasure to thank X. Bouju for reading the manuscript and his helpful comments. 
*m.seydou@cpmoh.u-bordeaux1.fr

†jp.aime@cpmoh.u-bordeaux1.fr

${ }^{1}$ R. S. Ruoff, D. Qian, and W. K. Liu, C. R. Phys. 4, 993 (2003).

${ }^{2}$ A. López-Bezanilla, F. Triozon, S. Latil, X. Blase, and S. Roche, Nano Lett. 9, 940 (2009).

${ }^{3}$ R. Voggu, S. Pal, S. K. Pati, and C. N. R. Rao, J. Phys.: Condens. Matter 20, 215211 (2008)

${ }^{4}$ B. Das, R. Voggu, C. S. Routa, and C. N. R. Rao, PhysChemComm 10, 5155 (2008).

${ }^{5}$ J. S. Bunch, T. N. Rhodin, and P. L. McEuen, Nanotechnology 15, S76 (2004)

${ }^{6}$ J. Martinez, T. D. Yuzvinsky, A. M. Fennimore, A. Z. R. Garcia, and C. Bustamante, Nanotechnology 16, 2493 (2005).

${ }^{7}$ E. S. Snow, P. M. Campbell, and J. P. Novak, Appl. Phys. Lett. 80, 2002 (2002).

${ }^{8}$ J. Charlier, X. Gonze, and J.-P. Michenaud, Europhys. Lett. 28, 403 (1994).

${ }^{9}$ Y. Baskin and L. Mayer, Phys. Rev. 100, 544 (1955).

${ }^{10}$ R. Nicklow, W. Wakabayashi, and H. G. Smith, Phys. Rev. B 5, 4951 (1972).

${ }^{11}$ L. A. Girifalco and R. A. Ladd, J. Chem. Phys. 25, 693 (1956).

${ }^{12}$ L. X. Benedict, N. G. Chopra, M. L. Cohen, A. Zettl, S. G. Louie, and V. H. Crespi, Chem. Phys. Lett. 286, 490 (1998).

${ }^{13}$ R. Zacharia, H. Ulbricht, and T. Hertel, Phys. Rev. B 69, 155406 (2004).

${ }^{14}$ A. Kis, G. Csanyi, J. Salvetat, T. Lee, E. Couteau, A. Kulik, W. Benoit, J. Brugger, and L. Forro, Nature Mater. 3, 153 (2004).

${ }^{15}$ S. B. Trickey, F. Muller-Plathe, G. H. F. Diercksen, and J. C. Boettger, Phys. Rev. B 45, 4460 (1992).

${ }^{16}$ H. J. F. Jansen and A. J. Freeman, Phys. Rev. B 35, 8207 (1987).

${ }^{17}$ M. C. Schabel and J. L. Martins, Phys. Rev. B 46, 7185 (1992).

${ }^{18}$ L. A. Girifalco and M. Hodak, Phys. Rev. B 65, 125404 (2002).

${ }^{19}$ Á. Szabados, L. P. Biró, and P. R. Surján, Phys. Rev. B 73, 195404 (2006).

${ }^{20}$ J.-C. Charlier and J.-P. Michenaud, Phys. Rev. Lett. 70, 1858 (1993).

${ }^{21}$ A. Ghosh, K. S. Subrahmanyam, K. S. Krishna, S. Datta, A. Govindaraj, S. K. Pati, and C. N. R. Rao, J. Phys. Chem. C 112, 15704 (2008).

${ }^{22}$ N. Varghese, U. Mogera, A. Govindaraj, A. Das, P. K. Maiti, A. K. Sood, and C. N. R. Rao, ChemPhysChem 10, 206 (2009).

${ }^{23}$ A. Hocquet and M. Langgard, J. Mol. Model. 4, 94 (1998).

${ }^{24}$ S. Ianelli, M. Nardelli, D. Belletti, B. Jamart-Gregoire, S. Mercier-Girardot, and P. Caubere, Acta Crystallogr., Sect. C: Cryst. Struct. Commun. 52, 1530 (1996).

${ }^{25}$ T. S. Balaban, A. T. Balaban, S. Foroc, and H.-J. Lindnerc, ARKIVOC 2002 (i), 1 (2002).
${ }^{26}$ K. Kim and K. Paek, Bull. Korean Chem. Soc. 24, 1375 (2003).

${ }^{27}$ G. S. Palagina and S. P. Sidorenko, Comput. Proteomics i, 188 (2002)

${ }^{28}$ S. De Feyter, A. Gesquire, F. D. Schryver, C. Meiners, M. Sieffert, and K. Mllen, Langmuir 16, 9887 (2000).

${ }^{29}$ A. Riemann and B. Nelson, Langmuir 25, 4522 (2009).

${ }^{30}$ V. A. Basiuk and M. Maria, J. Nanosci. Nanotechnol. 8, 259 (2008).

${ }^{31}$ J. Buchoux, J.-P. Aimé, R. Boisgard, C. V. Nguyen, L. Buchaillot, and S. Marsaudon, Nanotechnology 20, 475701 (2009).

${ }^{32}$ N. Allinger, J. Am. Chem. Soc. 99, 8127 (1977).

${ }^{33}$ N. Allinger, X. Zhou, and J. Bergsma, J. Mol. Struct.: THEOCHEM 312, 69 (1994).

${ }^{34}$ Computational Chemistry, Hyperchem Manual (Hypercube, Ontario, Canada, 1994).

${ }^{35}$ NANOTUBE MODELER, Manual, JCristalSoft: 2005-2006.

${ }^{36}$ S. L. Mayo, B. D. Olafson, and W. A. Goddard, J. Phys. Chem. 94, 8897 (1990).

${ }^{37}$ T. Hertel, R. E. Walkup, and P. Avouris, Phys. Rev. B 58, 13870 (1998).

${ }^{38}$ Y. J. Dappe, M. A. Basanta, F. Flores, and J. Ortega, Phys. Rev. B 74, 205434 (2006).

${ }^{39}$ D. P. DiVincenzo, E. J. Mele, and N. A. W. Holzwarth, Phys. Rev. B 27, 2458 (1983).

${ }^{40}$ H. Beyer, M. Muller, and T. Schimmel, Appl. Phys. A: Mater. Sci. Process. 68, 163 (1999).

${ }^{41}$ A. N. Kolmogorov and V. H. Crespi, Phys. Rev. B 71, 235415 (2005).

${ }^{42}$ H. Ruuska and T. A. Pakkanen, J. Phys. Chem. B 105, 9541 (2001).

${ }^{43}$ P. A. Gravil, M. Devel, P. Lambin, X. Bouju, C. Girard, and A. A. Lucas, Phys. Rev. B 53, 1622 (1996).

${ }^{44}$ Y. J. Dappe, J. Ortega, and F. Flores, Phys. Rev. B 79, 165409 (2009).

${ }^{45}$ M. Seydou, Y. Dappe, X. Bouju, S. Marsaudon, and J. P. Aimé (unpublished).

${ }^{46}$ A. Kis, K. Jensen, S. Aloni, W. Mickelson, and A. Zettl, Phys. Rev. Lett. 97, 025501 (2006).

${ }^{47}$ L. Marty, A. Iaia, M. Faucher, V. Bouchiat, C. Naud, M. Chaumont, T. Fournier, and A. Bonnot, Thin Solid Films 501, 299 (2006).

${ }^{48}$ L. Marty, V. Bouchiat, C. Naud, M. Chaumont, T. Fournier, and A. Bonnot, Nano Lett. 3, 1115 (2003).

${ }^{49}$ C. Nguyen, K. Chao, R. Stevens, D. Delzeit, A. Cassel, J. Harper, J. Han, and M. Meyyapan, Nanotechnology 12, 363 (2001). 